\title{
On theory of second harmonic generation in 2D nonlinear photonic crystals with arbitrary structures
}

\author{
V.E. Eshniyazov ${ }^{1}$, B. Kh. Eshchanov ${ }^{1}$, D. B. Yusupov², Q. Ya. Ergashev², U. K. Sapaevi,* \\ ${ }^{1}$ Department of Physics, National University of Uzbekistan, named after Mirzo Ulugbek, \\ 100095, Tashkent, Uzbekistan \\ ${ }^{2}$ Tashkent State Technical University, named after Abu Raikhan Beruni, \\ Universitetskya ul. 2, 100095, Tashkent \\ 1*usapaev@gmail.com
}

PACS 42.65.Ky

DOI 10.17586/2220-8054-2015-6-6-779-785

Second harmonic generation in 2D nonlinear photonic crystals based on rectangular symmetry with rectangular motifs has been analyzed theoretically. An approximate solution of the spectral response for the second harmonic generation in any designed 2D nonlinear photonic crystal is obtained within the un-depleted pump approximation. Rapid calculation of the temporal profile of multidirectional second harmonic pulse in such nonlinear lattices has been also shown.

Keywords: 2D nonlinear photonic crystals, second harmonic generation, frequency conversion, quasi-phase matching, arbitrary domain structures, linearly chirped domain structures, randomly sized domain structures.

Received: 1 November 2015

\section{Introduction}

One of the fundamental problems of nonlinear optics and laser physics is the creation of laser radiation in specific regions of a spectrum, where direct laser emission is either inefficient or impossible. In this regard, frequency convertors, based on nonlinear photonic crystals (NPC) are of great interest in practice. The technique of significant importance is quasi-phase matching, based on NPC, where real phase matching does not occur, but high conversion efficiencies are nevertheless obtained in a crystal where the sign (or strength) of the nonlinearity varies periodically. This type of periodic nonlinearity variation can be achieved by e.g. periodic poling. For the first time, this technique was developed by N. Bloembergen [1]. Since the first report in 1961, quasi-phase matched interactions in NPC have developed into widely used tools for the generation of coherent radiation. While more than five decades old, this field is still rapidly developing today.

In the last few years, 2D NPC, proposed by V. Berger [2] have given new possibilities for frequency convertors, since such lattices have several advantages in comparison with their 1D counterparts. For instance, they can be used to convert multiple frequencies with different directions from a single wave-length [3,4] or get high conversion efficiency [5], for simultaneous wavelength interchange [6], for all optical deflection and splitting realization [7], for realizing parametric solitons with purely nonlinear origin [8] and so on [9]. Relatively recently, an analytical solution was proposed to calculate the second harmonic intensity in rectangular 2D lattices for the nonlinear Raman-Nath diffraction process in the un- depleted pump approximation [10]. 


\section{Statement of the problem}

It is well known that 1D NPC with randomly sized domains have been the subject of intense studies due to possibilities of controlling spectral [11] or temporal profiles of generated harmonic, in particular, second harmonic generation (SHG) in the un-depleted [12] and depleted pump regimes [13-15]. It has been shown that harmonic generation with desired (e.g., amplitude and phase) parameters can be obtained by "exactly" designing 1D NPC. Pulse shaping technique via NPC with extremely short domains was also studied previously [16] for backward SHG energy conversion in the non-stationary regime. For this purpose and other tasks of frequency conversion problems, it is necessary to develop mathematical methods for analyzing spectral and temporal responses of 2D NPC at given three-wave interactions.

For the first time, the design of a SHG spectral response with any required profile in 1D NPC was developed in [11], where authors demonstrated this using a simulated annealing algorithm. Any amplitude and phase profile of second harmonic (SH) pulse in the undepleted pump approximation was theoretically showed in [12]. It is necessary to note that this problem was also solved for the case of the depleted pump approximation using an effective numerical method using simulated annealing algorithm [13].

Most previous works have only focused on the 1 D NPC, not on the 2 D NPC with destroyed domain structures. We believe that for a solution to this kind of problem, it is necessary to develop a rapid numerical or analytical method for analyzing 2D NPC with any arbitrary domain structures.

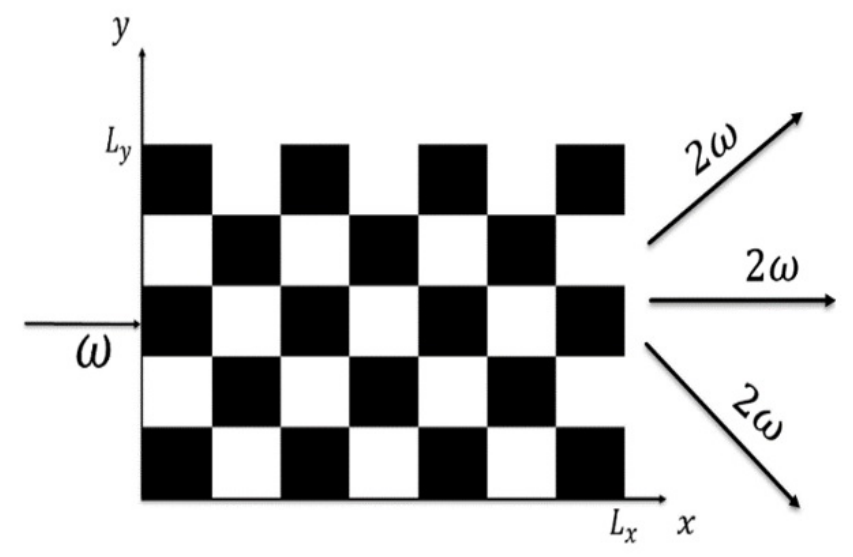

FIG. 1. Schematic picture of a $2 \mathrm{D} \chi^{(2)}$ crystal. NPC has rectangular symmetry with rectangular motifs, where $\chi^{(2)}$ has positive sign in black motifs, while in white- negative

\section{The concept of problem decision}

Every nonlinear crystal with second order nonlinearity has spectral bandwidth for chosen three-wave interaction, which is an important parameter during the generation of short laser pulses. This parameter is dependent on crystal length and/or temperature in "normal" quadratic nonlinear optical crystals and is very difficult to increase it for the generation of short laser pulses. For one's turn, NPC's spectral bandwidth is flexible and can be increased by changing domain sizes on various configurations. In our opinion, previous 
works on NPC with no "standard" (i.e., arbitrary sized) domain structures have been limited for only 1D NPC [11-15].

In the present work, we developed a rapid method for exactly calculating spectral and temporal response of SHG in 2D NPC with arbitrary structures. We focus on 2D NPC of rectangular symmetry with a rectangular motif.

\section{Realization of the concept}

The SHG process in 2D NPC is described by the following integral equation in the undepleted pump approximation $[2,9,17]$ (see Fig.1):

$$
E^{2 \omega}(\Delta k)=-i \alpha\left(E^{\omega}\right)^{2} \int_{0}^{L_{y}} \int_{0}^{L_{x}} d y d x \exp (-i \Delta \mathbf{k} \times \mathbf{r}) d(\mathbf{r})
$$

where, $\Delta \mathbf{k}=\mathbf{k}^{2 \omega}-2 \mathbf{k}^{\omega}$-is the phase mismatch of the interacting fields, $\alpha=\frac{2 \omega^{2} d_{33}}{k^{2 \omega} c^{2} L_{y}}, d_{33}-$ the effective coefficient of nonlinear susceptibility NPC, $E^{\omega}, \omega$-respectively, the complex amplitude and frequency of the fundamental wave, $d(\mathbf{r})$ is a sign of second order nonlinearity which could be 1 or -1 depending on motif positions; $L_{x}$ and $L_{y}$ are length and width(see Fig. 1).

Taking into account the sign change of the $d(\mathbf{r})$ function, the integral part of the equation (1) can be rewritten by the following way:

$$
E^{2 \omega}(\Delta k)=-i \alpha\left(E^{\omega}\right)^{2} \sum_{m=1}^{M} \sum_{n=1}^{N}(-1)^{m-n} \int_{B_{m-1}}^{B_{m}} d y \int_{A_{n-1}}^{A_{n}} d x \exp \left(-i \cdot\left(\Delta k_{x} x+\Delta k_{y} y\right)\right) .
$$

Furthermore, one can get the following solution (see also in $[10,11])$ :

$E^{2 \omega}(\Delta k)=-i \alpha\left(E^{\omega}\right)^{2} \sum_{m=1}^{M} \sum_{n=1}^{N}(-1)^{m-n} \cdot \frac{e^{-i \Delta k_{x} A_{n}}-e^{-i \Delta k_{x} A_{n-1}}}{\Delta k_{x}} \cdot \frac{e^{-i \Delta k_{y} B_{m}}-e^{-i \Delta k_{y} B_{m-1}}}{\Delta k_{y}}$.

$$
\begin{aligned}
& A=\left[a_{1} ; a_{1}+a_{2} ; a_{1}+a_{2}+a_{3} ; \ldots ; a_{1}+a_{2}+\ldots+a_{N}\right] \\
& B=\left[b_{1} ; b_{1}+b_{2} ; b_{1}+b_{2}+b_{3} ; \ldots ; b_{1}+b_{2}+\ldots+b_{M}\right]
\end{aligned}
$$

Another crucial feature of SHG in 2D NPC is multidirectional generation and their symmetry. The phase mismatch of the interacting fundamental frequency and $\mathrm{SH}$ can be calculated by using the following formula (see Fig. 2) [2,16]:

$$
\Delta k=-2 k^{\omega} \cdot \cos \phi+k^{2 \omega} \cdot \sqrt{1-\left(\frac{2 k^{\omega}}{k^{2 \omega}}\right)^{2} \cdot \sin ^{2} \phi}
$$

Using (4) and (3), spectral response of SHG can be calculated.

Figure 3 shows the results of numerical (dashed) and analytical (solid) calculations of SH's spectral response in 2D NPC of rectangular symmetry with rectangular motif depending on the angle of rotation. To demonstrate it, we looked at a realistic case: 2D NPC with $N=100$ and $M=100$ based on lithium niobate by $1550 \mathrm{~nm}$ fundamental frequency emission. For this case, we calculated:

$$
a_{1}=a_{2}=\ldots=a_{N}=b_{1}=b_{2}=\ldots=b_{M} \approx 9.4 \mu \mathrm{m}
$$

for $\mathbf{G}(1,0)$ lattices wave-vector $[2,16]$. As is seen from the figure, numerical and analytical results correspond to each other with high accuracy for any configuration of NPC. Analytical 


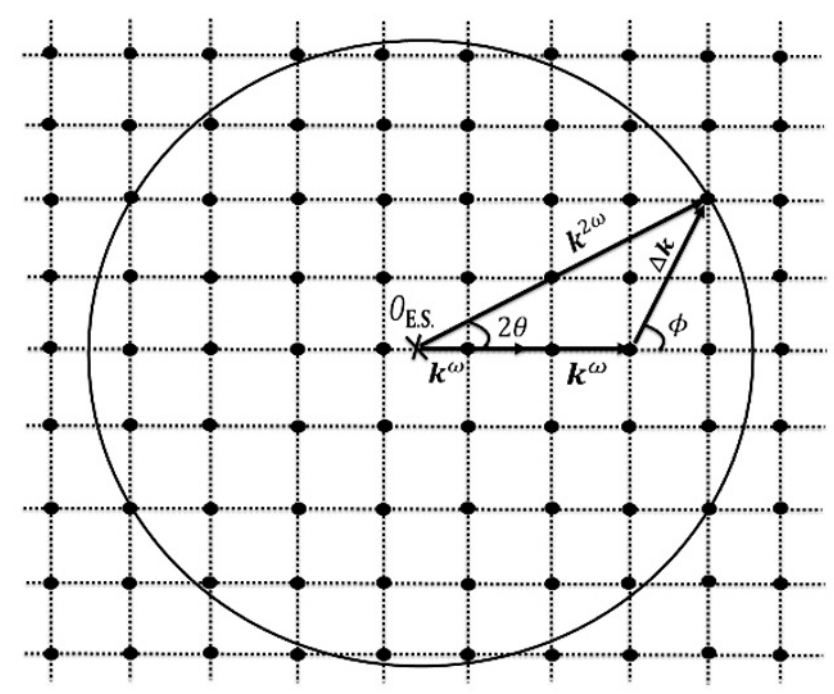

FIG. 2. Phase mismatch schema for SHG in 2D NPC

calculation took much less time than numerical one during calculation. (In our case it was 25 times less, due to each motif being divided by 5 steps on $x$-axis and $y$-axis coordinates for numerical calculation). Figure 3 (1) shows the uniform NPC, where all domains have the same height and width (i.e., $\sim 9.4 \mu \mathrm{m}$ ). As it is seen here $\mathbf{G}(1,1)$, the quasi-phase matching order is higher than $\mathbf{G}(1,0)$, although the opposite would be expected. From the above equation (3), it is easily seen that the maximum value of $\left|E^{2 \omega}(\Delta k)\right|$ is achieved when $\left|e^{-i \Delta k_{x} A_{n}}-e^{-i \Delta k_{x} A_{n-1}}\right|$ and $\left|e^{-i \Delta k_{y} B_{m}}-e^{-i \Delta k_{y} B_{m-1}}\right|$ takes their highest values, i.e. \pm 2 . Therefore, the phase mismatch vector $\Delta k\left(\frac{\pi u}{a} ; \frac{\pi v}{b}\right)$ is considered as the best frequency conversion condition where $u$ and $v$ are odd integers, whereas they are even integers SHG will be shut down. So SHG occurs more intensely at $\phi=45^{\circ}(u=1, v=1)$ than at $\phi=0^{\circ}$ $(u=1, v=0)$.

Figure $3(2)$ shows results, when 2D NPC has linear chirp on the x-axis coordinate only such as from the first motif $(9.4-9.4 \cdot 0.05) \mu \mathrm{m}$ the last motif $(9.4+9.4 \cdot 0.05) \mu \mathrm{m}$ on both coordinates. As we expected, there we can see the spectral broadening of the $\mathrm{SH}$ wave in both directions at an angle $\phi=45^{\circ}$. This is a well-known effect in 1D NPC. Figure 3 (3) shows results when $2 \mathrm{D}$ NPC has randomly-sized motifs in both the $\mathrm{x}$ - and $\mathrm{y}$-axis directions. In this case, it appears $(1,0)$ quasi-phase matching order due to decreasing phase mismatch between the fundamental frequency and SH waves. To calculate the last case, we randomly changed sizes of every motif on both directions by $5 \%$ of their original sizes. By increasing the factor of random sizes, we were able to get overlapping all three peaks. In a realistic case, this can allow one to obtain broader broadband bandwidth of spectrum of 2D NPC in all directions spatially.

The main advantage of the analytical method is its quickness and its utility if we concentrate 2D NPC with very large numbers of motifs. This is especially important, as the design of this type of nonlinear optical medium could require a large number of calculations (e.g. around 1000 or more motifs).

Finally, we studied the possibility of calculating the temporal profile of a SH pulse at different angles. For this, we used the previously-developed convolution method [18]. Within this method, equation (3) can be used as a spectral response of the nonlinear medium, i.e. in our case 2D NPC and by multiplying it to the spectral profile of the square of the fundamental 

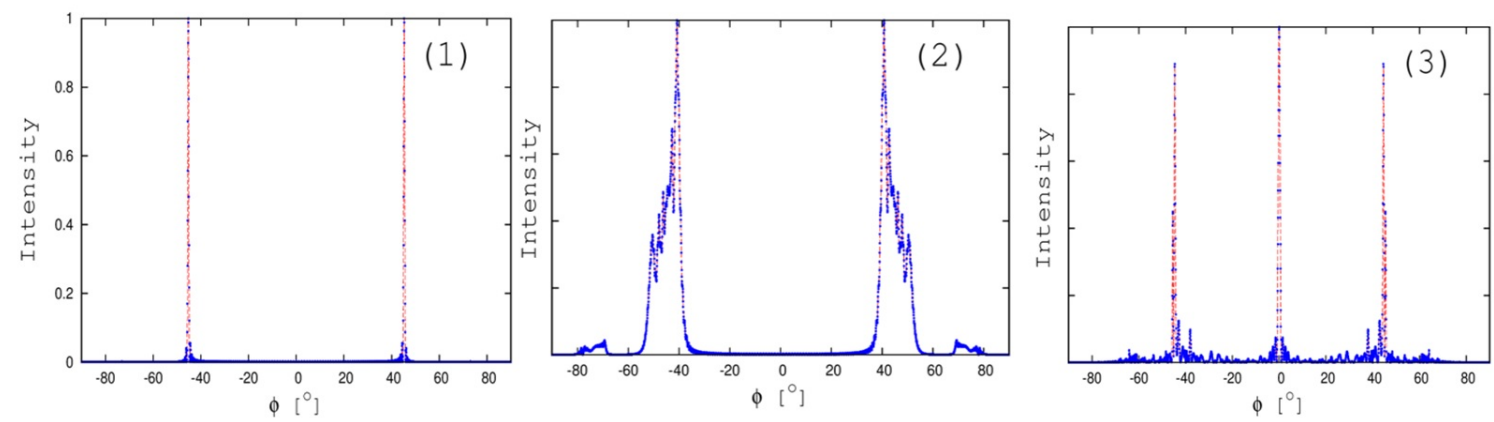

FIG. 3. Comparison of results of analytical results, based on equation (3) with numerical method for different realistic cases of SHG: (1) uniform 2D NPC; (2) linearly chirped 2D NPC; (3) randomly sized 2D NPC

pulse. The convolution method can be written by this formula:

$$
A_{s h}(t)=\operatorname{BFT}\left(E_{s h}(\Omega) * F F T\left(A^{2}(t)\right)\right),
$$

here BFT and FFT are the backward and forward Fourier transform, respectively; $A(t)$ is the temporal profile of input pulse. Equation (5) takes into account the dispersion of the nonlinear medium.

Figure 4 show results of the calculation, based on equation (5), where the input pulse was taken to be a 50 femtosecond at FWHM and the spectral response of 2D NPC was taken approximately at an angle of $\sim 45^{\circ}$. The spectral response of the nonlinear lattices was divided by the same number of the steps for the temporal profile of the fundamental pulse from $42^{\circ}$ to $48^{\circ}$. One can see from the figure that the temporal profile of the SH pulse is increased due to a group velocity mismatch between the fundamental and SH pulses. (By increasing numbers of motifs on both coordinates, we were able to receive broader SH pulse relatively to the fundamental one, because of influence of the group velocity mismatch. This data is not presented here).

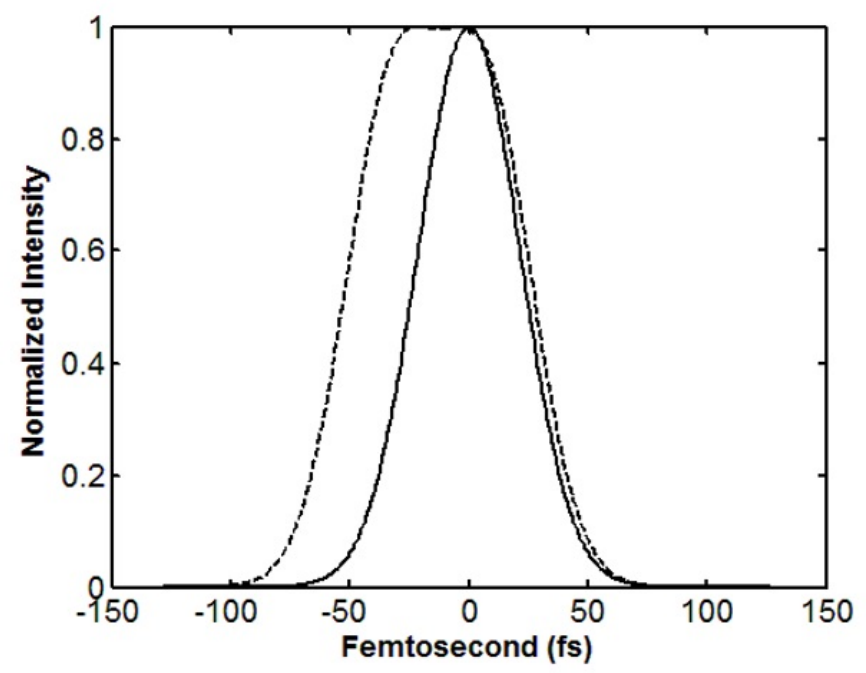

FIG. 4. Results of rapid calculation (5): temporal profiles of input (solid) and second harmonic (dashed) pulses at around angle $\sim 45^{\circ}$ 


\section{Conclusion}

In conclusion, we have theoretically studied SHG in 2D nonlinear photonic crystals, based on rectangular symmetry with rectangular motif. We obtained an approximation solution for the spectral response of SHG in any designed 2D nonlinear photonic crystals within the un-depleted pump approximation. We also showed a calculation of the temporal profile of SH pulse in such nonlinear lattices. We focused on a realistic case of the frequency doubling in 2D NPC of lithium niobate by a femtosecond fundamental pulse with fundamental wavelength of $1550 \mathrm{~nm}$. Theoretical methods of this work can be used for other frequency conversion processes and another type and motifs of 2D NPC. This method can also be applied to multiple harmonic generation in 2D NPC, simultaneously. The latter is possible, if there is no pump depletion of the fundamental frequency. Finally, the presented mathematical method, due to its quickness, can be used to design 2D NPC with arbitrarily sized structures using various optimization algorithms.

\section{Acknowledgments}

D. B. Yusupov and U.K. Sapaev thank A.S. Chirkin and A.M.Vyunishev for enlightening discussions. This work was supported by the Basic Research Foundation of the Ministry of Higher and Secondary Special Education of the Republic of Uzbekistan Under contract N F2-49.

\section{References}

[1] J.A. Armstrong, N. Bloembergen, D. Ducing, P.S. Pershan. Interaction between light waves in a nonlinear dielectric. Phys. Rev., 1962, 127, P. 1918.

[2] V. Berger. Nonlinear photonic crystals. Phys. Rev. Lett., 1998, 81, P. 4136.

[3] N. G. R. Broderick, R. T. Brafalean, T. M. Monro, D. J. Richardson, C. M. de Sterke. Temperature and wavelength tuning of second-, third-, and fourth-harmonic generation in a two-dimensional hexagonally poled nonlinear crystal. J. Opt. Soc. Am. B, 2002, 19, P. 2263.

[4] S. Saltiel, Y. S. Kivshar. Phase matching in nonlinear photonic crystals, Opt. Lett., 2000, 25, P. 1204.

[5] N.G.R. Broderick, G.W. Ross, H.L. Offerhaus, D.J. Richardson, D.C. Hanna: Hexagonally poled lithium niobate: a two-dimensional nonlinear photonic crystal. Phys. Rev. Lett., 2000, 84, P. 4345.

[6] A. Chowdhury, C. Staus, B.F. Boland, T.F. Kuech, L. McCaughan, Experimental demonstration of 1535-1555 nm simultaneous optical wavelength interchange with a nonlinear photonic crystal. Opt. Lett., 2001, 26, P. 1353.

[7] S.M. Saltiel, Y.S. Kivshar: All - optical deflection and splitting by second - order cascading. Opt. Lett., 2002, 27, P. 921.

[8] K.Gallo, A.Pasquazi, S.Stivala, G.Assanto. Parametric Solitons in Two-Dimensional Lattices of Purely Nonlinear Origin. Phys. Rev. Lett., 2008, 100, P. 053901.

[9] A.Arie, N.Voloch. Periodic, quasi-periodic, and random quadratic nonlinear photonic crystals. Laser 86 Photon. Rev., 2010, 4, P. 355.

[10] A.M.Vyunishev, A.S.Chirkin. Multiple quasi-phase-matching in nonlinear Raman-Nath diffraction. Opt. Lett., 2015, 40, P. 1314.

[11] D. T. Reid. Engineered quasi - phase - matching for second - harmoni cgeneration. Journal of optics A - pure and applied optics, 2003, 5(4), P. 97.

[12] U. K. Sapaev, D. T. Reid. General second - harmonic pulse shaping in grating - engineered quasi phase - matched nonlinear crystals. Optics Express, 2005, 13, P. 3264.

[13] U. K. Sapaev, G. Assanto. Femtosecond pulse synthesis by efficient second - harmonic generation in engineered quasi phase matching gratings. Optics Express, 2007, 15, P. 7448.

[14] U. K. Sapaev. Optimum Formation of the Response of Aperiodic Nonlinear Crystals in the Process of Second Harmonic Generation. Opt. and Spect., 2007, 102, P. 939.

[15] D.B.Yusupov, U. K. Sapaev. Nonlinear Optics in Photonic Crystals. Tashkent, FAN, 2012, p. 128. 
[16] U.K.Sapaev, D.B. Yusupov, A.A. Sherniyzov, A.A. Uzakov. Theory of backward second-harmonic generation of short laser pulses in periodically and a periodically poled nonlinear crystals. Journal of Russian Laser Research, 2012, 33, P. 103.

[17] S.M. Russel, P.E. Powers, M.J. Missey, K.L. Schepler. Broadband mid - infrared generation with two dimensional quasi-phase-matched structures. IEEE J. Quant. Electron, 2001, 37, P. 877.

[18] M.A. Arbore, O. Marco, M.M. Fejer. Pulse compression during second - harmonic generation in a periodic quasi-phase - matching gratings. Opt. Lett., 1997, 22, P. 438. 